\title{
Polystyrene Encapsulated SERS Tags as Promising Standard Tools: Simple and Universal in Synthesis; Highly Sensitive and Ultrastable for Bioimaging
}

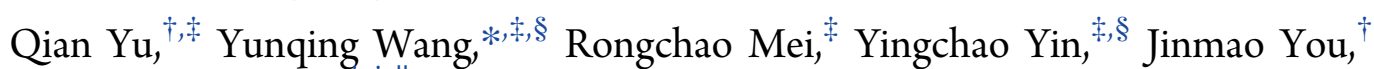
and Lingxin Chen $*,+, \|_{10}$

\author{
${ }^{\dagger}$ Department of Chemistry and Chemical Engineering, Qufu Normal University, Qufu 273165, China \\ ${ }^{\ddagger}$ CAS Key Laboratory of Coastal Environmental Processes and Ecological Remediation, Yantai Institute of Coastal Zone Research, \\ Chinese Academy of Sciences, Yantai 264003, China \\ ${ }^{\S}$ School of Pharmacy, Collaborative Innovation Center of Advanced Drug Delivery System and Biotech Drugs in Universities of \\ Shandong, Key Laboratory of Molecular Pharmacology and Drug Evaluation (Yantai University), Ministry of Education, Yantai \\ University, Yantai 264005, China \\ "Laboratory for Marine Biology and Biotechnology, Pilot National Laboratory for Marine Science and Technology, Qingdao \\ 266237, China
}

Supporting Information

\begin{abstract}
Surface coating determined the sensitivity and stability of surfaceenhanced Raman scattering (SERS) tags in bioanalysis. The reported various coatings suffered from the drawbacks of a lack of rigidity, stability, or synthesis versatility. Herein, we demonstrated robust polystyrene (PS) coated SERS tags that could be prepared by an easy and universal approach. Taking advantages of biocompatible, transparent, compact properties of PS shell, the coated tags showed satisfactory sensitivity, biocompatibility, and superior structural stability in cell and in vivo imaging applications. More importantly, the PS coating strategy allowed for the encapsulation of SERS tags encoded with not only thiolated but also nonthiolated Raman reporters without loss of sensitivity, as exemplified in the synthesis of 9 different resonant dye-encoded tags. Moreover, the coating of SERS tags with various kinds of substrates was achieved via the same standard protocol. Comparing with widespread silica coated tags, the PS coated ones were more stable in harsh conditions and had an easily expanded ultrasensitive (resonant) tags library with much lower cost (no need of expensive sulfhydryl/isothiocyano reporters with limited types), illustrating great promise as standard analytical tools of commercialized value for bioanalysis, medical diagnostics, and environmental science studies.

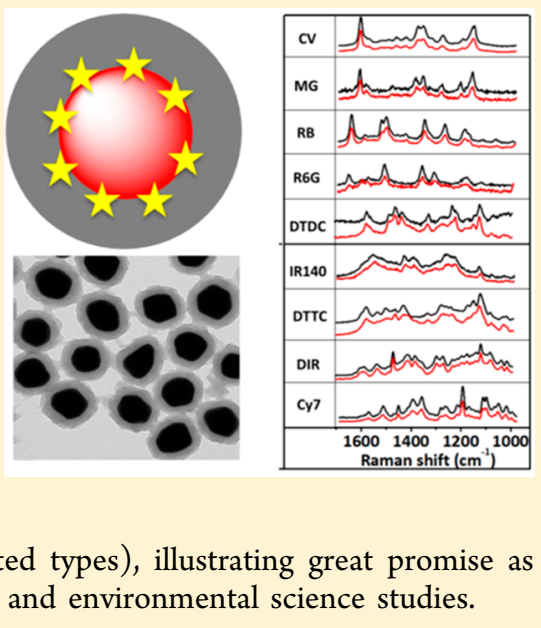

\begin{abstract}
Urface-enhanced Raman scattering (SERS) tags have $\checkmark$ attracted much attention for bioimaging analysis due to high sensitivity, multiplex labeling ability, and satisfactory biocompatibility. ${ }^{1}$ A bare SERS tag is composed of two main parts, i.e., a noble metal nanoparticle (NP) as a Raman signal enhancement substrate and reporter molecules for the generation of characteristic Raman peaks. The colloidal and optical stabilities of bare tags are often compromised by structural alteration or ligand dissociation of Raman reporters in complicated biological environments. Therefore, additional surface coating materials, as an additional component of SERS tags, are extensively screened to encapsulate the bare tags to overcome these problems. Up to now, many kinds of coating materials including biomolecules (protein, ${ }^{2-4}$ lipid $^{5-9}$ ), "soft" polymers (SH-PEG, ${ }^{10,11}$ polydopamine ${ }^{12-14}$ ), inorganic shells (silica, ${ }^{15-21}$ titania $^{22}$ ), and carbon materials (mesoporous carbon, ${ }^{23}$ graphitic capsule ${ }^{24-27}$ ) as well as metallic layers (such as those within gap-enhanced Raman tags) ${ }^{28-32}$ have been reported.
\end{abstract}

There are several concerns for the selection of coating materials. The first is biocompatibility, which is a basic requirement for producing probes for biological applications. The second is the permeability and structural stability. SERS tags may encounter many different biological conditions, such as those rich in protein, detergent, and salt, with a broad $\mathrm{pH}$ range (from $\mathrm{pH} 1$ in stomach to $\mathrm{pH} \mathrm{8-9}$ in intestine), and long-term exposure in physiological temperature $\left(37^{\circ} \mathrm{C}\right)$ during the in vivo imaging application. An ideal coating should be firm enough to protect the inner NPs and Raman reporters in all these harsh conditions. Mesoporous inorganic shells and linear polymer coatings cannot effectively prevent the penetration of interference molecules. Lipid bilayer coating is sensitive to many kinds of stimulus (detergent, heat, lipid

Received: January 3, 2019

Accepted: March 18, 2019

Published: March 18, 2019 
exchange) due to its soft and fluidic nature. ${ }^{33}$ Solid silica shell is impermeable; however, it tends to degrade in weak basic media $^{34,35}$ and cannot stand for long-term storage. ${ }^{36}$

The third concern is the transparency of the coating material. Silica and polymer are satisfactory in this aspect. Despite ultrathin graphitic shells also being transparent, it will introduce intrinsic Raman D and G bands as background signals of SERS tags. ${ }^{27,37}$ Because of the strong light adsorption property, polydopamine ${ }^{12}$ and carbon $\operatorname{shell}^{23}$ dramatically reduce the signals of SERS tags with the increasing thickness of the shells.

The fourth one is the convenience and versatility of synthesis. The synthesis of silica coating required multiple processes, including the unavoidable modification of vitreophilic agents (i.e., PVP, ${ }^{38}$ MUA, ${ }^{17}$ and SH-PEG, ${ }^{39}$ etc.) on noble metal NPs in advance. Moreover, the $\mathrm{pH}$ of the coating medium must be adjusted to alkaline for the hydrolysis of TEOS, which greatly weakens the affinity of positively charged Raman reporters to the NPs. As a result, the signal intensity of the coated tags sharply decreased. Owing to this problem, silica coated SERS tags tended to involve nonresonant, thio-/ isothiocyano-containing Raman reporters. The encapsulation of varied, nonthiolated but resonant molecules to increase the sensitivity and expand the "SERS tag library" could hardly be realized.

In recent years, SERS tags had a blooming development but most attention had been paid on the nanosubstrates and Raman reporters. From the aspect of coating, silica and $\mathrm{SH}$ PEG are generally regarded as standard materials and seldom doubted. In view of the above discussions, there were still many unsolved fundamental problems on this issue, and the development of novel coating materials and methods for SERS tags were still in great demand.

We speculated that polystyrene (PS) was advantageous as coating material for SERS tags. (1) From the aspect of biosafety, fluorescent PS NPs had been widely applied in cell/ in vivo imaging as standard labeling tools for their good biocompatibility. ${ }^{40,41}$ (2) As a material that can be used to produce optical devices, PS has high transparency with light transmittance of about $90 \%$ and refractive index of $1.59 .^{42}$ This feature is helpful to reduce signal loss from the coating and maximally preserve the signal of SERS tags. (3) The structural compactness and stability of PS is also prominent. ${ }^{36}$ It can effectively prevent interfering molecules from entering the SERS tag shell and protect signal stability of the tags. Once tags were developed into commercially available probes, a long-term storage period could also be expected. Up to now, SERS tags prepared by depositing metal NPs on the surface of PS sphere templates had been proposed, ${ }^{43-47}$ but those with PS coating shells had never been reported.

Herein, we prepared PS coated SERS tags for the first time via an easy and universal approach. Taking advantage of biocompatible, transparent, rigid properties of PS shell, the tags showed satisfactory sensitivity, monodispersity, biocompatibility, and superior structural stability. More importantly, the PS coating was a versatile strategy that allowed for encapsulation of both thiolated and nonthiolated dye-encoded tags, greatly increased the diversity, and lowered the cost of the ultrasensitive resonant SERS (SERRS) tags. Besides, the encapsulation of SERS tags with substrates of various shapes and capping ligands, including hydroxylamine capped gold nanospheres, HEPES coated gold nanoflowers, and CTAB coated gold nanorods, was achieved via the same protocol. The
PS coated SERS tags exhibited strong and stable signals in cell and in vivo imaging applications, illustrating great potential as alternative standard tools for the widespread silica coated tags in bioanalysis and medical diagnostics.

\section{EXPERIMENTAL SECTION}

Materials. Chloroauric acid $\left(\mathrm{HAuCl}_{4}\right)$, hydroxylamine hydrochloride $\left(\mathrm{NH}_{2} \mathrm{OH} \cdot \mathrm{HCl}\right)$, sodium hydroxide $(\mathrm{NaOH})$, sodium chloride $(\mathrm{NaCl})$, sodium bromide $(\mathrm{NaBr})$, anhydrous sodium sulfate $\left(\mathrm{NaSO}_{4}\right)$, silver nitrate $\left(\mathrm{AgNO}_{3}\right)$, L-ascorbic acid (AA), crystal violet (CV), malachite green (MG), rhodamine $\mathrm{B}(\mathrm{RB})$, rhodamine 6G (R6G), tetraethyl orthosilicate (TEOS), styrene (St), hydrochloric acid $(\mathrm{HCl})$, ammonia ammonium hydroxide $\left(\mathrm{NH}_{3} \cdot \mathrm{H}_{2} \mathrm{O}\right)$, and ethanol were purchased from Sinopharm Chemical Reagent Co. Ltd. Sodium citrate, sodium borohydride $\left(\mathrm{NaBH}_{4}\right)$, and poly(vinylpyrrolidone) (PVP) were obtained from Aladdin. Glutathione (GSH), cetyltrimethylammonium bromide (CTAB), cetyltrimethylammonium chlorine (CTAC), 3,3'diethylthiatricarbocyanine iodide (DTTC), 3,3'-diethylthiadicarbocyanine iodine (DTDC), Cy7, 4-nitrothiophenol (4NT), and IR140 were purchased from Sigma-Aldrich. 1,1'Dioctadecyl-3,3,3',3'-tetramethylindotricarbocyanine iodide (DIR) was obtained from AAT Bioquest. Divinylbenzene (DVB) and 2,2-azobis(2-methylpropyl)dihydrochloride (AIBA) were purchased from Macklin. 4-(2-Hydroxyethyl)-1piperazineethanesulfonic acid (HEPES) was obtained from Life Science Products. Lipoid E80 was purchased from Lipoid GmbH. 11-Mercaptoundecanoic acid (MUA) was obtained from J\&K Scientific. SH-PEG was obtained from Shanghai Yare Biotech. CKK-8-enhanced solution was obtained from Meilunbio. Dulbecco's modified Eagle's medium (DMEM), penicillin-streptomycin solution, fetal bovine serum, and trypsin-ethylenediaminetetraacetic acid solution were purchased from Thermo Scientific. Deionized water was used in all the experiments $\left(18.2 \mathrm{M} \Omega \mathrm{cm}^{-1}\right)$.

Characterization. Transmission electron microscopy (TEM) images were obtained on a JEM-1400 transmission electron microscope (JEOL, Japan). Scanning electron microscopy (SEM) images were acquired on an S-4800 field emission scanning electron microscope (Hitachi, Japan). The UV-vis absorption spectra were recorded on a Thermo Scientific NanoDrop 2000/2000C spectrophotometer. The $\zeta$ potential and dynamic light scattering (DLS) size were measured on a Zetasizer NanoZS90 (Malvern Instruments, U.K.). The SERS spectra were recorded on a DXR Raman microscope (Thermo Scientific, USA). A 632.8 or $780 \mathrm{~nm}$ laser was focused by a $10 \times$ and a $50 \times$ microscope objective for the sample solution, cell or mouse measurements.

Synthesis of $110 \mathrm{~nm}$ Gold Nanospheres (AuNSs). AuNSs was synthesized via a seed growth method. ${ }^{48}$ Briefly, $1.0 \mathrm{~mL}$ of sodium citrate ( $1 \mathrm{wt} \%$ ) was quickly added into the boiling solution of $100 \mathrm{~mL}$ of $\mathrm{HAuCl}_{4}(0.01$ wt \%) under stirring and refluxing. The mixture solution continued boiling for $30 \mathrm{~min}$, and then the solution was cool to room temperature. The NPs in this solution were used as Au seeds. A $4.0 \mathrm{~mL}$ aliquot of the seed solution and $0.9 \mathrm{~mL}$ of sodium citrate ( $1 \mathrm{wt} \%$ ) were added to $53 \mathrm{~mL}$ of water, followed by addition of $0.9 \mathrm{~mL}$ of $\mathrm{HAuCl}_{4}$ (1 wt \%) and stirring for $8 \mathrm{~min}$. Then, $1.4 \mathrm{~mL}$ of $\mathrm{NH}_{2} \mathrm{OH} \cdot \mathrm{HCl}(10 \mathrm{mM})$ was added dropwise with continuous stirring. The reaction should be complete after $5 \mathrm{~min}$ and stirring was continued for $1 \mathrm{~h}$ at room temperature. 
Synthesis of Bare SERS Tags. Bare SERS tags were obtained by mixing $20 \mathrm{~mL}$ of AuNS solution and $200 \mu \mathrm{L}$ of Raman reporter solution for $0.5 \mathrm{~h}$. The final concentration was $10^{-6} \mathrm{M}$ for NT, IR140, RB, DTTC DIR, and Cy7 and $10^{-7} \mathrm{M}$ for $\mathrm{CV}, \mathrm{MG}, \mathrm{DTDC}$, and R6G.

Synthesis of PS Coated SERS Tags. First, $19.5 \mathrm{~mL}$ of water and $82.5 \mathrm{~mL}$ of ethanol were added to a mixture of purified St $(0.95 \mathrm{~mL})$, DVB $(0.05 \mathrm{~mL})$, and PVP $(300 \mathrm{mg})$ in a $250 \mathrm{~mL}$, two-necked flask equipped with a reflux condenser and a Teflon coated magnetic stirring bar. The reaction mixture was stirred at $70{ }^{\circ} \mathrm{C}$ for $1 \mathrm{~h}$ under a nitrogen atmosphere. Then, $3 \mathrm{~mL}$ of an aqueous AIBA solution (1.7 wt $\%$ ) was added to the flask. Eight min later, $15 \mathrm{~mL}$ of bare SERS tag solution was introduced into the reaction mixture. The reaction was continued with heating at $70{ }^{\circ} \mathrm{C}$ for another $18 \mathrm{~h}$ under a nitrogen atmosphere. The PS coated SERS tags were washed 5 times by being centrifuged in water to remove the residual reactants and free PS NPs.

Cell Culture and Imaging. A549 cells were grown as a monolayer in a humidified incubator at $37{ }^{\circ} \mathrm{C}$ in air $/ \mathrm{CO}_{2}$ (95:5). The medium was DMEM that was supplemented with $10 \%$ fetal bovine serum. For all experiments, the cells were washed with phosphate-buffered saline (PBS) and harvested by using trypsin. All cells were finally resuspended in a fresh medium before plating. For cell imaging, the A549 cells $(2 \times$ $10^{4}$ ) were seeded onto glass coverslips in a 24-well plate with $500 \mu \mathrm{L}$ of DMEM medium and cultured for $12 \mathrm{~h}$ to allow the cells to attach. Then, $100 \mu \mathrm{L}$ of each SERS tag $(40 \mu \mathrm{g} / \mathrm{mL})$ was added into the cell culture. After incubation for different times $(3,6,12$, and $24 \mathrm{~h})$, the cell monolayer on the coverslip was repeatedly washed with PBS to remove the remaining particles and then sealed with a glass microscope slide. The cells were fixed on glass coverslips before imaging. The observations were performed by Raman microscopy, and the SERS mapping could be performed at the same time.

MTT Assay. In a 96-well plate, $5 \times 10^{3}$ A549 cells were plated and incubated for $12 \mathrm{~h}$ to allow the cells to attach. Then $\mathrm{Au}$ NPs SERS tags were added to the cells with different concentrations, and the cells were incubated at $37{ }^{\circ} \mathrm{C}$ for another $24 \mathrm{~h}$. Cell viability of the tags was evaluated by using an MTT assay.

In Vivo SERS Detection. The in vivo detection of the Au star@Cy7@PS SERS tags was tested in three administration ways. (1) The tag solution was subcutaneously injected at a leg of a mouse, and the time-dependent SERS signal at the injection site was in situ monitored. (2) The tag solution was intravenously injected into a mouse through the tail vein. Afterward, the signals from the main organs were recorded through skin at different time intervals. Fifteen h later, the mouse was executed and SERS signals of liver, spleen, lung, heart, and kidney were measured after stripping off the overlying skin. (3) The tag solution was given to a mouse via intragastric administration route. The signal from stomach was monitored 15 min postadministration. Five h later, the mouse was executed and the SERS signal from ex vivo intestine was measured. In order to examine the possibility of the tags to pass through the intestinal tract barrier, another mouse was subjected to 2 consecutive days' intragastric administration, and then the SERS signal of tail vein blood was recorded. For each way $200 \mu \mathrm{L}$ of the tags $(350 \mu \mathrm{g} / \mathrm{mL})$ was administrated, followed by the detection of the SERS spectrum from specific sites or organs using Raman microscopy with a $780 \mathrm{~nm}$ laser $(150 \mathrm{~mW})$.

\section{RESULTS AND DISCUSSION}

Morphology and Spectral Properties of PS Coated SERS Tags. Figure 1A illustrated the synthesis procedure of
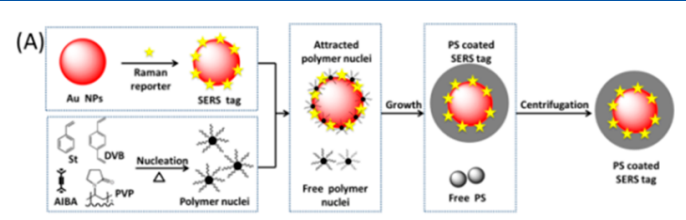

(B)
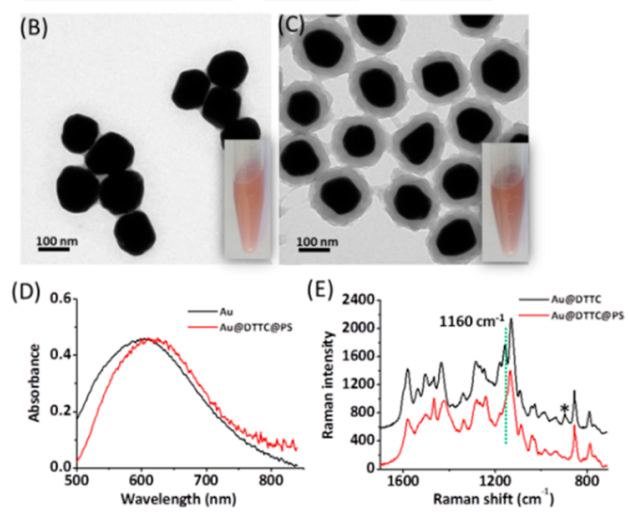

Figure 1. (A) Schematic illustration of synthesis procedure of PS coated SERS tags. TEM image of AuNS cores (B) and AuNS@ DTTC@PS SERS tags (C). The inset pictures showed the sample solutions. (D) UV-vis spectra of AuNS cores and AuNS@DTTC@ PS SERS tags. (E) Raman spectra of SERS tag before and after PS shell coating. The peak marked with asterisk $\left(890 \mathrm{~cm}^{-1}\right)$ was from ethanol for preparing DTTC solution.

PS coated SERS tags. First, bare SERS tags were prepared by adsorbing Raman reporter molecules on the surface of AuNSs. Then the bare tags were added to the mixture of a polymerization solution containing St as the monomer, PVP as the emulsifier, DVB as the cross-linking agent, and AIBA as the initiator. After the polymerization proceeded for $8 \mathrm{~min}$, bare SERS tags were added to the polymerization solution. A large amount of PVP capped free polymer nuclei quickly attached on and stabilized the bare tags, assuring the monodispersity of the tags in ethanol-rich medium, without interfering adsorption of the Raman reporters. With further growth of the attached polymer nuclei, highly cross-linked and compact PS shell in situ formed on the surface of the tags, resulting in the formation of PS coated SERS tags. Free PS NPs were also produced, but they could be easily separated from the tags via centrifugation due to the large density distinction between the two NPs. The prepolymerization of St was a critical step, without which the morphology of PS coated tags was irregular and a lot of large PS aggregates formed (Supporting Information Figure S1).

Panels B and C of Figure 1 illustrated TEM images of the core AuNSs and AuNS@DTTC@PS SERS tags, respectively. The AuNSs were encapsulated inside PS shells with a thickness around $35 \mathrm{~nm}$. The particle size distribution of the tags was uniform according to dynamic light scattering (DLS) results (Figure S2). A small fraction of NP clusters (about $10.6 \%$ dimer and $2.8 \%$ trimer counted by $280 \mathrm{NPs}$ ) formed probably during the NP and Raman reporter mixing process, which could be effectively separated via a further low speed centrifugation (Figure S3). $\zeta$ potential of the tags was 26.7 $\mathrm{mV}$, which made the dispersion of the colloid as good as the AuNS core solution $(-5.69 \mathrm{mV})$. UV-visible spectra of Au NS 
cores and AuNS@DTTC@PS SERS tags were illustrated in Figure 1D. The SPR wavelength of the Au NS core red-shifted from 598 to $635 \mathrm{~nm}$ after the coating process, which was due to the increasing refractive index environment (from 1.33 of water to 1.59 of PS) and the slight aggregation of the AuNS cores. The PS coated tags perfectly preserved SERS signal intensity of the bare tags (Figure 1E), indicating the coating protocol did not affect the structure and NP-attachment of Raman reporters. It should be noted that the peak at 1160 $\mathrm{cm}^{-1}$ (a benzene ring $\mathrm{C}-\mathrm{C}$ stretching vibration of DTTC) decreased and merged with its neighboring peak after PS coating, probably owing to specific interaction of this molecular domain with the surrounding PS polymer chain. ${ }^{49}$

Effect of PS Thickness on SERS Intensity. The effect of PS thickness on SERS intensity was studied. TEM images in Figure $2 \mathrm{~A}-\mathrm{D}$ showed that the thickness of the tags could be
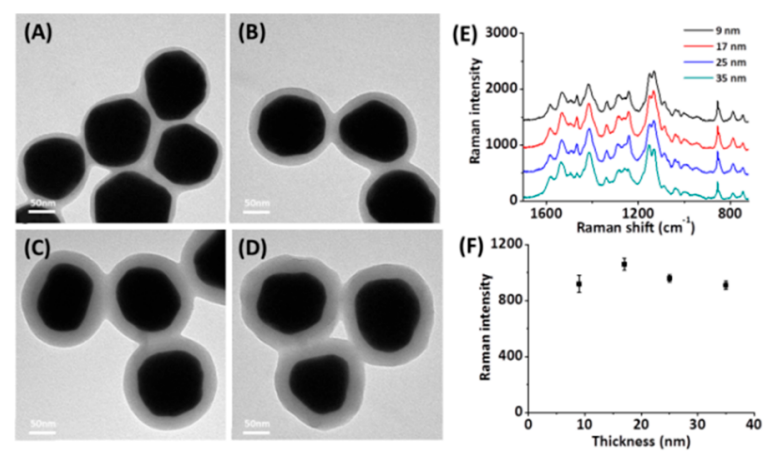

Figure 2. TEM images of AuNS@DTTC@PS SERS tags with polymerization reaction time of $10 \mathrm{~min}(\mathrm{~A}), 1 \mathrm{~h}(\mathrm{~B}), 5 \mathrm{~h} \mathrm{(C)}$, and 18 $\mathrm{h}$ (D). The shell thicknesses were about 9, 17, 25, and $35 \mathrm{~nm}$, respectively. (E) Raman signal of PS coated SERS tags of different shell thickness. (F) SERS signal intensity of $1130 \mathrm{~cm}^{-1}$ peak of the four samples. The data are presented as the mean intensity with standard deviation $(n=3)$.

tuned from $9 \mathrm{~nm}$ at $10 \mathrm{~min}$ to $35 \mathrm{~nm}$ at $18 \mathrm{~h}$. Taking advantage of the transparency of PS, the increasing thickness of the PS shell had little effect on the Raman signal intensity of the tags (Figure 2E,F) and the relative standard deviation of the signal intensity for samples with four different thicknesses was only $7.1 \%$, indicating that PS coating was superior to the polydopamine coating and carbon shell coating that could dramatically shield the signal of the tags. ${ }^{12,23}$ Much thinner PS layer $(2-3 \mathrm{~nm})$ could also be obtained by collecting the product with a shorter reaction time (Figure S4); however, these tag samples were not further characterized considering the probably weakened robustness for bioimaging applications.

Encapsulation of SERS Tags with Different Kinds of Raman Reporters and SERS Substrates. The ease of the coating procedure rendered the encapsulation of SERS tags with various kinds of Raman reporters including not only thiolated ones which could strongly attach on gold surface via $\mathrm{Au}-\mathrm{S}$ chemical bond but also nonthiolated molecules with weak affinity via weak electrostatic interaction. Figure $3 \mathrm{~A}$ showed Raman spectra of 10 PS coated SERS tags; among them 9 spectra were from nonthiolated "resonant" Raman reporter molecules. The signals of all bare SERS tags were perfectly preserved after PS coating, regardless of the type of reporters. For comparison, we also tried to encapsulate the bare tags inside silica shells via a widespread Stöber method. ${ }^{17}$ However, the coating was hard to proceed with because when

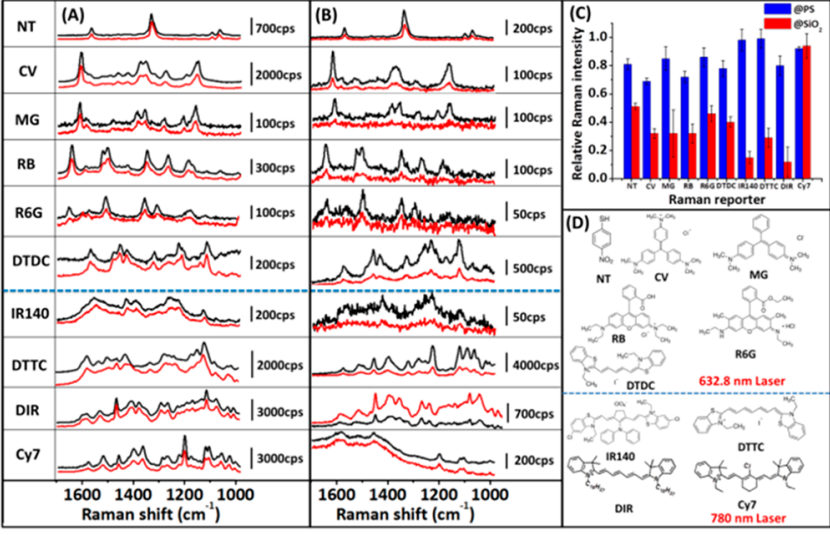

Figure 3. (A) Raman spectra of SERS tags adsorbing different kinds of Raman reporters before (black line) and after PS coating (red line). (B) Raman spectra of SERS tags adsorbing different kinds of Raman reporters before (black line) and after $\mathrm{pH}$ adjustment for silica coating (red line). (C) Signal preservation comparison for PS and silica coating methods using different Raman reporters. The data are presented as the mean value of three measurements with standard deviation. (D) Chemical structures of the Raman reporters.

the $\mathrm{pH}$ of the coating medium was adjusted to alkaline for hydrolysis of TEOS, the signal of 9 bare tags (except for Cy7) dramatically decreased to between $16 \%$ and $50 \%$ of the initial values (Figure $3 \mathrm{~B}, \mathrm{C}$ ), relying on the different cross-sections and affinity to the meal surface of the Raman reporters (Figure 3D). The decrease in signal could be attributed to two reasons. (1) The $\mathrm{pH}$ change would alter the structure of the Raman reporter and made its electronic configuration from a resonant form to a nonresonant form. (2) The increased $\mathrm{pH}$ weakened the electrostatic interaction between negative charged AuNSs and positive charged reporters, resulting in detachment and aggregation of the reporters (Figure S5). This unavoidable problem made the type of reporter restricting in thio- or isothiocyano-containing molecules. Despite few nonthiolated molecules also being reported, ${ }^{15,19,20}$ the sensitivity of the silica coated tags was too much lost comparied with the bare ones. The PS coating strategy avoided the $\mathrm{pH}$ adjustment and showed no interference on the signal of the tags. It broadened the reporter library and benefitted the preparation of a large set of resonant reporter coded, sensitive SERS tags for multiplex labeling.

Furthermore, we investigated the possibility to coat other SERS nanosubstrates, including citrate capped $\mathrm{Au} \mathrm{NP}$, lipid bilayer capped core-satellite (C-S) NP, CTAB coated gold nanorods (Au NR), and HEPES coated gold nanoflowers ( $\mathrm{Au}$ NFs) via the same protocol. Figure 4 showed the TEM images and SERS spectra of the products, which indicated that this method was versatile on coating NPs with different morphologies and capping ligands and surface charges. Besides, TEM images and UV-vis absorption spectra (Figure S6) proved that PS coating conditions $\left(70{ }^{\circ} \mathrm{C}\right)$ did not cause morphology change of multibranched cores such as Au NFs and nanostars, which might reshape toward spherical NPs at high temperature.

Stability of PS Coated SERS Tag. PS is resistant to various alkalis, salts, and certain acids (such as sulfuric acid, phosphoric acid, boric acid, and hydrochloric acid with a mass fraction of $10 \%$ to $30 \%$ aqueous solutions. The impermeability and structural stability of PS coating had been demonstrated in previous works. It could provide hydrophobic environment to 


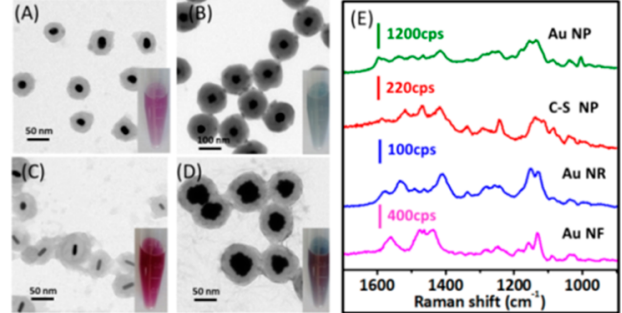

Figure 4. TEM images of PS coated tags with different cores. (A) Citrate capped Au NP, (B) lipid bilayer capped C-S NP, (C) CTAB coated Au NR, and (D) HEPES coated Au NF. The inset pictures showed the sample solutions. (E) SERS spectra of the tags with DTTC as Raman reporter.

protect the lanthanide complexes ${ }^{50}$ or quantum dots ${ }^{51}$ from being quenched by outside species presented in water. In our work, PS coated SERS tags showed excellent signal stability in various biologically related environments (Figure 5A). No
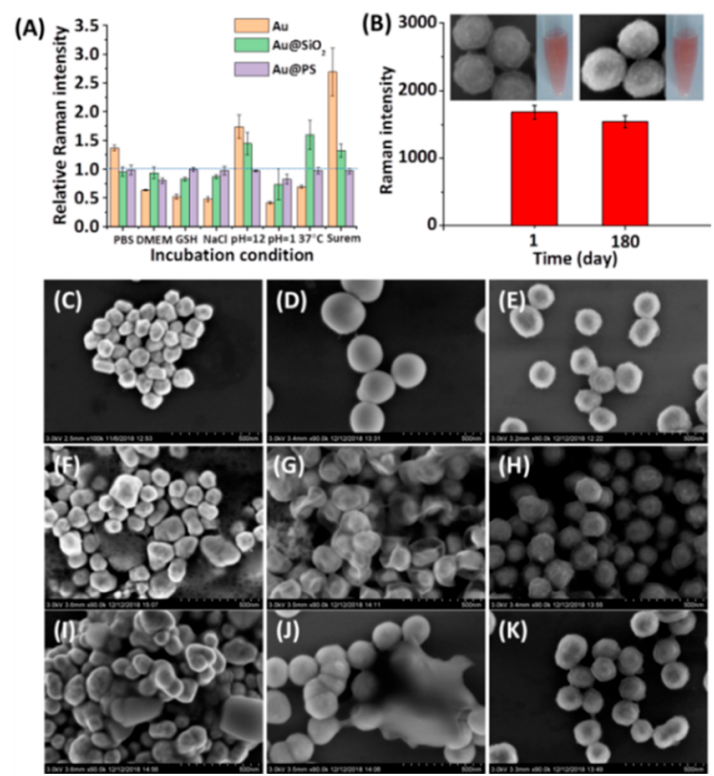

Figure 5. (A) SERS signal variations of bare AuNS-DTTC tags, silica coated tags, and PS coated tags under different biologically related incubation conditions for $30 \mathrm{~min}$. Relative Raman intensity indicated the ratio of the peak intensity at $1133 \mathrm{~cm}^{-1}$ value of the tags before and after incubation. (B) Signal intensity, morphology, and solution color of AuNS@DTTC@PS SERS tags before and after 180 days' storage. Raman intensity data were presented as the mean intensity of $1130 \mathrm{~cm}^{-1}$ peak with standard deviation $(n=3)$. SEM images of AuNS-DTTC tags (C, F, I), silica coated tags (D, G, J), and PS coated tags $(\mathrm{E}, \mathrm{H}, \mathrm{K})$ without treatment $(\mathrm{C}, \mathrm{D}, \mathrm{E})$, incubated in 37 ${ }^{\circ} \mathrm{C}, \mathrm{pH} 7.4 \mathrm{PBS}(\mathrm{F}, \mathrm{G}, \mathrm{H})$ and in $\mathrm{pH} 12$ aqueous solution at $25^{\circ} \mathrm{C}(\mathrm{I}$, $\mathrm{J}, \mathrm{K})$ for $24 \mathrm{~h}$.

significant variation in SERS intensity of PS coated tags was observed in PBS ( $\mathrm{pH}$ 7.4), DMEM, serum, glutathione (GSH, $1 \mathrm{mM}), \mathrm{NaCl}(1 \mathrm{mM})$, basic and acidic water $(\mathrm{pH} 12$ and $\mathrm{pH}$ $1)$, and $37{ }^{\circ} \mathrm{C}$ incubation conditions. By contrast, the signal intensity of bare tags underwent a sharp increasing or decreasing in different conditions due to aggregation of NPs or detachment of Raman reporters. For silica coated tags, slight agglomeration occurred under $\mathrm{pH} 12$ and $37{ }^{\circ} \mathrm{C}$ conditions and the Raman signal was apparently enhanced, probably owing to the dissolution of silica shell. Considering the need for continuous, long-term monitoring, the photostability of the PS coated tags was also investigated, by comparing with that of SH-PEG coated tags. As shown in Figure S7, the signal of PEG coated tags decreased dramatically to $48 \%$ of its initial value due to the reporter detachment from the tags, while SERS signal remained essentially unchanged for PS shell protected tags. The PS coated tags also demonstrated satisfactory longterm storage structural (Figure S8) and signal stability. After 180 days' storage the PS shell was still compact and the signal intensity maintained around $92 \%$ of the initial value (Figure 5B).

The superior signal stability came from the ideal structural stability of PS coating. To prove this, the three tags (Figure 5 $\mathrm{C}, \mathrm{D}, \mathrm{E}$ ) were dispersed in $\mathrm{pH} 7.4 \mathrm{PBS}$ at $37{ }^{\circ} \mathrm{C}$ and $\mathrm{pH} 12$ water for $24 \mathrm{~h}$, respectively. Bare tags aggregated and fused to some extent in both conditions (Figure 5 F,I). For PBS treated silica tags, the solid NPs with rigid coating dramatically changed to interesting rattle structures with soft, wrinkled shell because of a selective silica etching process ${ }^{35}$ (Figure 5G). Differently, the incubation in basic atmosphere $(\mathrm{pH} 12)$ tended to induce the dissolution of silica from the outer surface, indicated by the fusion of the coated NPs and the randomly spread pasty material (dissolved sodium silicate) on the NPs (Figure 5J). As expected, PS coating held great advantage from the aspect of structural stability. It remained intact in both harsh conditions (Figure $5 \mathrm{H}, \mathrm{K}$ ), thus offering a stable environment for the tags and assuring the signal stability.

Cell Imaging. In view of the bioimaging applications, the cytotoxicity of the PS coated tags was evaluated via the MTT assay. Figure S9 showed that cell viability was larger than $80 \%$ at the highest dose of the tags $(100 \mu \mathrm{g} / \mathrm{mL})$. The value was $95.5 \%$ at the tag concentration used for cellular imaging (40 $\mu \mathrm{g} / \mathrm{mL}$ ), indicating the biocompatibility was satisfactory for potential biological applications. Then, the living cell imaging experiments were performed by using DTTC- and CVencoded tags by utilizing 780 and $632.8 \mathrm{~nm}$ laser irradiation, respectively. Both tags were observed to be taken up by the cells and characteristic peaks could be measured inside the cells (Figure 6A,B). To illustrate the signal protection effect of the PS shell inside living cells, we compared the imaging performance of nonthiolated reporter crystal violet (CV)encoded tags with and without PS coating. As shown in Figure 6C, the Raman signal of the bare AuNS@CV tags quickly decreased and nearly disappeared after $24 \mathrm{~h}$ in A549 cells. The signal loss was owing to the displacement of weak adsorbed $\mathrm{CV}$ by endogenous thiol-containing molecules. By contrast, the intensity of AuNS@CV@PS tags gradually increased because of the uptake by the cells, and a strong signal was maintained as the culture time extended to $24 \mathrm{~h}$ (Figure 6D). This signal stability was comparable with that of the AuNS@CV@SiO ${ }_{2}$ tag counterpart whose intracellular stability was well accepted (Figure S10).Therefore, it could be concluded that the PS shell effectively prevented the interference from cells, making the unstable, nonthiolated Raman reporter-coded SERS tag qualified for living cell imaging applications.

In Vivo Imaging. In vivo imaging potential of the PS coated SERS tags were demonstrated by three proof-ofconcept experiments, applying Au star@Cy7@PS tags which showed extraordinary sensitivity under $780 \mathrm{~nm}$ laser irradiation (Figure S11). First, the tag solution $(350 \mu \mathrm{g} / \mathrm{mL})$ was subcutaneously injected at a leg of a mouse and monitored the SERS signal with a $780 \mathrm{~nm}$ laser irradiation (Figure 7A). Shown in Figure $7 \mathrm{~B}, \mathrm{C}$ was a strong and characteristic SERS 

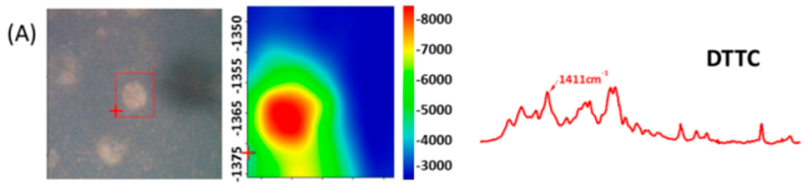

159201593015940
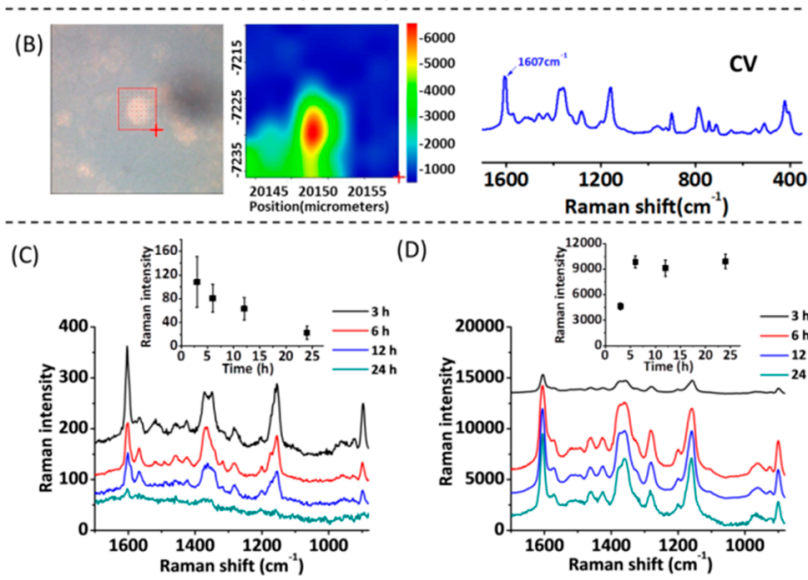

(D)

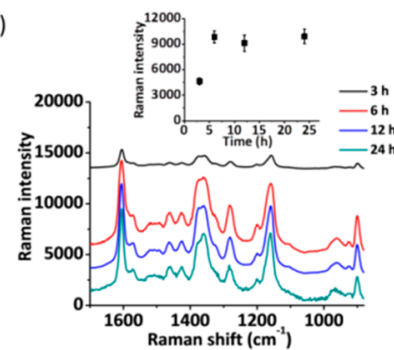

Figure 6. SERS imaging of the A549 cells using AuNS@DTTC@PS (A) and AuNS@CV@PS SERS tags (B) after incubation for $24 \mathrm{~h}$. The images were obtained. The spectra were extracted from the intracellular regions. Mean SERS spectra of bare AuNS@CV SERS tags (C) and AuNS@CV@PS SERS tags (D) at different time intervals after incubation with the cells. Each mean spectrum was plotted from 20 spectra from different cell positions. The insets showed intensity variations of the peak at $1607 \mathrm{~cm}^{-1}(n=20)$.

signal of the tag at the first $24 \mathrm{~h}$ at the injection region, whereas it decreased to about $1 / 3$ of the initial value at $50 \mathrm{~h}$ and was totally undetected after $70 \mathrm{~h}$, indicating the clearance of tag solution. Second, the tags were intravenously injected into a mouse through the tail vein. Characteristic peaks of the tag could be clearly observed from liver and spleen after $1 \mathrm{~h}$ from this living mouse through the skin (Figure 7D), indicating the in vivo, deep tissue imaging capability. After $15 \mathrm{~h}$, the mouse was executed, and the SERS signals from ex vivo main organs were recorded after stripping off the overlying skin. The liver, spleen, and lung showed strong signals, and a weak peak was measured from the heart and kidney (Figure 7E). These results provided preliminary distribution information on the tags in mouse. Third, to test the signal stability of the tags in the digestive system, the tag solution was intragastrically administrated to a mouse. A strong signal could be recorded from the stomach of the living mouse after $15 \mathrm{~min}$ (Figure 7F, up panel). The mouse was executed after $5 \mathrm{~h}$, followed by the SERS measurement of the digestive organs. Strong SERS signal could be observed from a specific section of small intestine, which not only provided the transportation location of the tags but also verified the tags withstand the harsh environments of the digestive system (low $\mathrm{pH}$ at stomach and weak basic, rich of digestive ferment at intestine). In order to examine the possibility of the tags to pass through intestinal tract barrier, a mouse was subjected to 2 consecutive days' intragastric administration of the tags, followed by the signal measurement from tail vein blood (Figure 7F, low panel). Apparent SERS signal was recorded in the blood, implying that the tags had crossed intestinal epithelial cells, entered the blood circulation and been taken to the whole body.

Micro-/nanoplastics as a novel environmental pollutant have drawn worldwide attention. It was important to elaborate the
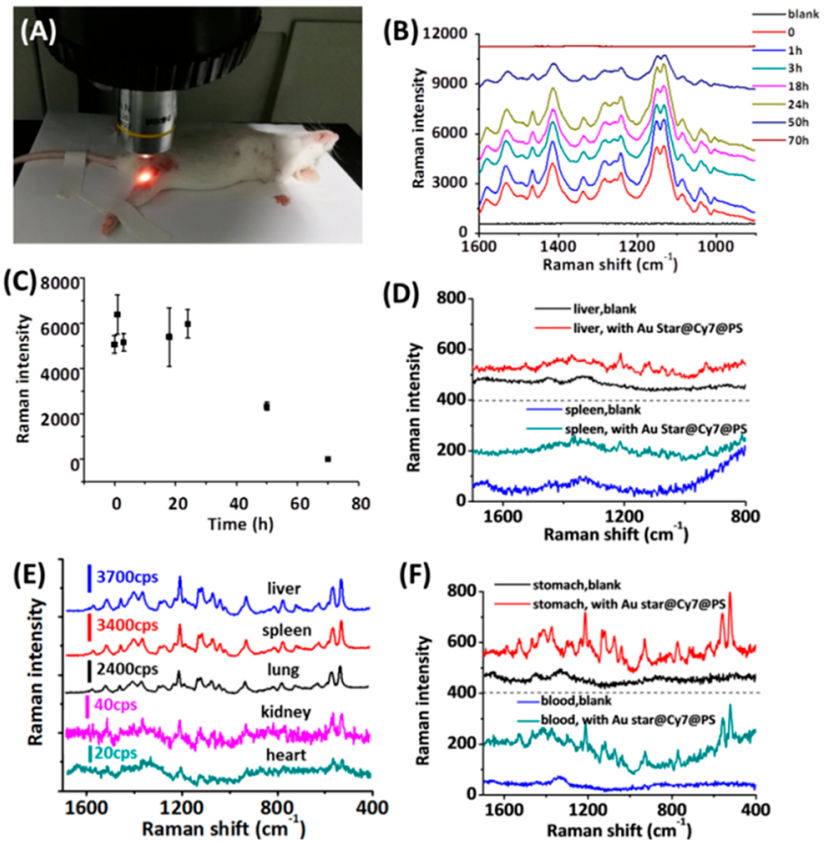

Figure 7. (A) In vivo SERS detection of a mouse subcutaneously injected at a leg. (B) SERS spectra from the injection site at different time intervals. (C) Time-dependent SERS tag signal intensity variation at the injection site of the mouse. The data are presented as the mean intensity at $1130 \mathrm{~cm}^{-1}$ with standard deviation $(n=3)$. (D) SERS spectra from liver (up panel) and spleen (low panel) of a living mouse $1 \mathrm{~h}$ postinjection through the tail vein. The blank spectra were recorded from the anatomical locations of liver and spleen of the mouse before tag injection. (E) SERS spectra from ex vivo main organs from the same mouse $15 \mathrm{~h}$ post intravenous injection. (F) Up panel, SERS spectra from stomach of a living mouse $15 \mathrm{~min}$ postintragastric administration recorded through skin. The blank spectrum was recorded from the anatomical location of the stomach of this mouse before tag administration. Low panel, spectrum collected from tail vein blood of a mouse treated with 2 consecutive days' intragastric administration of SERS tags. The blank spectrum was recorded from the blood of this mouse before tag administration.

in vivo behavior through imaging technology and then evaluate the safety risk on animals and human beings. From this point of view, PS coated tags could serve as labeled nanoplastics models and played an important role in the field of environmental sciences. Our preliminary results implied toxicity risk via an oral exposure of nanoplastics, and detailed works on this issue are still ongoing.

\section{CONCLUSIONS}

In summary, we developed a simple and universal approach for the synthesis of PS coated SERS tags. The tags exhibited satisfactory biocompatibility, sensitivity, and signal stability. An underlined advantage of the PS coating strategy was that it could encapsulate tags encoded with normal dyes without thiol groups without loss of the sensitivity. This paved the way for the expansion of stable and ultrasensitive resonant tag library in a simple and low cost manner. Furthermore, the bioimaging capability of PS coated SERS tags was demonstrated through proof-of-concept of living cell and in vivo imaging applications. The PS coated tags held great potential in preclinical diagnosis as standard probes and in environmental science as novel optical labeling nanoplastics models. 


\section{ASSOCIATED CONTENT}

\section{S Supporting Information}

The Supporting Information is available free of charge on the ACS Publications website at DOI: 10.1021/acs.analchem.9b00038.

Experimental details; SEM images; DLS data; TEM images; Color evolutions;signal decrease during TEOS hydrolysis; relative Raman intensities; MTT assay results; intracellular signals; SERS TEM images (PDF)

\section{AUTHOR INFORMATION}

\section{Corresponding Authors}

*E-mail: yqwang@yic.ac.cn (Y.W.).

*E-mail: lxchen@yic.ac.cn (L.C.).

\section{ORCID $\odot$}

Lingxin Chen: 0000-0002-3764-3515

Notes

The authors declare no competing financial interest.

\section{ACKNOWLEDGMENTS}

Financial support from the National Natural Science Foundation of China (Grants 81573393 and 21575159), the Youth Innovation Promotion Association CAS (Grant 2017256), and the Instrument Developing Project of the Chinese Academy of Sciences (Grant YZ201662) is gratefully acknowledged.

\section{REFERENCES}

(1) Wang, Y.; Yan, B.; Chen, L. Chem. Rev. 2013, 113, 1391-428.

(2) Niu, X.; Chen, H.; Wang, Y.; Wang, W.; Sun, X.; Chen, L. ACS Appl. Mater. Interfaces 2014, 6, 5152-5160.

(3) Li, J. R.; Wang, J.; Grewal, Y. S.; Howard, C. B.; Raftery, L. J.; Mahler, S.; Wang, Y. L.; Trau, M. Anal. Chem. 2018, 90, 1037710384.

(4) He, S.; Kyaw, Y. M. E.; Tan, E. K. M.; Bekale, L.; Kang, M. W. C.; Kim, S. S. Y.; Tan, I.; Lam, K. P.; Kah, J. C. Y. Anal. Chem. 2018, 90, 6071-6080.

(5) Lee, J. H.; Shin, Y.; Lee, W.; Whang, K.; Kim, D.; Lee, L. P.; Choi, J. W.; Kang, T. Sci. Adv. 2016, 2, e1601838-e1601838.

(6) Stewart, A. F.; Lee, A.; Ahmed, A.; Ip, S.; Kumacheva, E.; Walker, G. C. ACS Nano 2014, 8, 5462-5467.

(7) Tam, N. C.; McVeigh, P. Z.; MacDonald, T. D.; Farhadi, A.; Wilson, B. C.; Zheng, G. Bioconjugate Chem. 2012, 23, 1726-30.

(8) Ip, S.; MacLaughlin, C. M.; Gunari, N.; Walker, G. C. Langmuir 2011, 27, 7024-33.

(9) Mei, R. C.; Wang, Y. Q.; Liu, W. H.; Chen, L. X. ACS Appl. Mater. Interfaces 2018, 10, 23605-23616.

(10) Nguyen, C. T.; Nguyen, J. T.; Rutledge, S.; Zhang, J. N.; Wang, C.; Walker, G. C. Cancer Lett. 2010, 292, 91-97.

(11) von Maltzahn, G.; Centrone, A.; Park, J. H.; Ramanathan, R.; Sailor, M. J.; Hatton, T. A.; Bhatia, S. N. Adv. Mater. 2009, 21, 31753180 .

(12) Jiang, C. H.; Wang, Y.; Wang, J. W.; Song, W.; Lu, L. H. Biomaterials 2017, 114, 54-61.

(13) Li, J.; Liu, H. Q.; Rong, P. F.; Zhou, W.; Gao, X. H.; Liu, D. B. Nanoscale 2018, 10, 8292-8297.

(14) Sun, C. L.; Gao, M. X.; Zhang, X. M. Anal. Bioanal. Chem. 2017, 409, 4915-4926.

(15) Harmsen, S.; Huang, R.; Wall, M. A.; Karabeber, H.; Samii, J. M.; Spaliviero, M.; White, J. R.; Monette, S.; O'Connor, R.; Pitter, K. L.; Sastra, S. A.; Saborowski, M.; Holland, E. C.; Singer, S.; Olive, K. P.; Lowe, S. W.; Blasberg, R. G.; Kircher, M. F. Sci. Transl. Med. 2015, 7, 271 ra7.

(16) Liu, X. J.; Knauer, M.; Ivleva, N. P.; Niessner, R.; Haisch, C. Anal. Chem. 2010, 82, 441-446.
(17) Mir-Simon, B.; Reche-Perez, I.; Guerrini, L.; Pazos-Perez, N.; Alvarez-Puebla, R. A. Chem. Mater. 2015, 27, 950-958.

(18) Nayak, T. R.; Andreou, C.; Oseledchyk, A.; Marcus, W. D.; Wong, H. C.; Massagué, J.; Kircher, M. F. Nanoscale 2017, 9, 11101119.

(19) Oseledchyk, A.; Andreou, C.; Wall, M. A.; Kircher, M. F. ACS Nano 2017, 11, 1488-1497.

(20) Spaliviero, M.; Harmsen, S.; Huang, R.; Wall, M. A.; Andreou, C.; Eastham, J. A.; Touijer, K. A.; Scardino, P. T.; Kircher, M. F. Mol. Imaging Biol. 2016, 18, 677-85.

(21) Walters, C. M.; Pao, C.; Gagnon, B. P.; Zamecnik, C. R.; Walker, G. C. Adv. Mater. 2018, 30, 1705381.

(22) Zhang, W.; Wang, Y.; Sun, X.; Wang, W.; Chen, L. Nanoscale 2014, 6, 14514-14522.

(23) Shen, A. G.; Chen, L. F.; Xie, W.; Hu, J. C.; Zeng, A.; Richards, R.; Hu, J. M. Adv. Funct. Mater. 2010, 20, 969-975.

(24) Lin, D.; Qin, T.; Wang, Y.; Sun, X.; Chen, L. ACS Appl. Mater. Interfaces 2014, 6, 1320-9.

(25) Lai, X. F.; Zou, Y. X.; Wang, S. S.; Zheng, M. J.; Hu, X. X.; Liang, H.; Xu, Y. T.; Wang, X. W.; Ding, D.; Chen, L.; Chen, Z.; Tan, W. H. Anal. Chem. 2016, 88, 5385-5391.

(26) Song, Z. L.; Chen, Z.; Bian, X.; Zhou, L. Y.; Ding, D.; Liang, H.; Zou, Y. X.; Wang, S. S.; Chen, L.; Yang, C.; Zhang, X. B.; Tan, W. H. J. Am. Chem. Soc. 2014, 136, 13558-13561.

(27) Zou, Y. X.; Chen, L.; Song, Z. L.; Ding, D.; Chen, Y. Q.; Xu, Y. T.; Wang, S. S.; Lai, X. F.; Zhang, Y.; Sun, Y.; Chen, Z.; Tan, W. H. Nano Res. 2016, 9, 1418-1425.

(28) Lim, D.-K.; Jeon, K.-S.; Hwang, J.-H.; Kim, H.; Kwon, S.; Suh, Y. D.; Nam, J.-M. Nat. Nanotechnol. 2011, 6, 452-460.

(29) Lin, L.; Zapata, M.; Xiong, M.; Liu, Z.; Wang, S.; Xu, H.; Borisov, A. G.; Gu, H.; Nordlander, P.; Aizpurua, J.; Ye, J. Nano Lett. 2015, 15, 6419-6428.

(30) Kang, J. W.; So, P. T. C.; Dasari, R. R.; Lim, D.-K. Nano Lett. 2015, 15, 1766-1772.

(31) Zhang, Y.; Liu, Z.; Thackray, B. D.; Bao, Z.; Yin, X.; Shi, F.; Wu, J.; Ye, J.; Di, W. Small 2018, 14, 1801022.

(32) Zhang, Y.; Qiu, Y.; Lin, L.; Gu, H.; Xiao, Z.; Ye, J. ACS Appl. Mater. Interfaces 2017, 9, 3995-4005.

(33) Su, X.; Wang, Y.; Wang, W.; Sun, K.; Chen, L. ACS Appl. Mater. Interfaces 2016, 8, 10201-11.

(34) Hu, X. G.; Gao, X. H. ACS Nano 2010, 4, 6080-6086.

(35) Zhang, Q.; Zhang, T. R.; Ge, J. P.; Yin, Y. D. Nano Lett. 2008, 8, 2867-2871.

(36) Yang, D. P.; Cao, C.; Feng, W.; Huang, C. H.; Li, F. Y. J. Rare Earths 2018, 36, 113-118.

(37) Zhang, L.; Liu, F.; Zou, Y. X.; Hu, X. X.; Huang, S. Q.; Xu, Y. T.; Zhang, L. F.; Dong, Q.; Liu, Z. K.; Chen, L.; Chen, Z.; Tan, W. H. Anal. Chem. 2018, 90, 11183-11187.

(38) Kustner, B.; Gellner, M.; Schutz, M.; Schoppler, F.; Marx, A.; Strobel, P.; Adam, P.; Schmuck, C.; Schlucker, S. Angew. Chem., Int. Ed. 2009, 48, 1950-1953.

(39) Fernandez-Lopez, C.; Mateo-Mateo, C.; Alvarez-Puebla, R. A.; Perez-Juste, J.; Pastoriza-Santos, I.; Liz-Marzan, L. M. Langmuir 2009, $25,13894-13899$.

(40) Wang, T.; Wang, L.; Li, X.; Hu, X.; Han, Y.; Luo, Y.; Wang, Z.; Li, Q.; Aldalbahi, A.; Wang, L.; Song, S.; Fan, C.; Zhao, Y.; Wang, M.; Chen, N. ACS Appl. Mater. Interfaces 2017, 9, 18619-18625.

(41) Frohlich, E. Curr. Drug Metab. 2013, 14, 976-988.

(42) Zhao, Y.; Wang, J. S.; Mao, G. Z. Opt. Lett. 2005, 30, 18851887.

(43) Guo, I. W.; Pekcevik, I. C.; Wang, M. C. P.; Pilapil, B. K.; Gates, B. D. Chem. Commun. 2014, 50, 8157-8160.

(44) Lee, J.-H.; Mahmoud, M. A.; Sitterle, V. B.; Sitterle, J. J.; Meredith, J. C. Chem. Mater. 2009, 21, 5654-5663.

(45) Mir-Simon, B.; Morla-Folch, J.; Gisbert-Quilis, P.; Pazos-Perez, N.; Xie, H.-n.; Bastús, N. G.; Puntes, V.; Alvarez-Puebla, R. A.; Guerrini, L. J. Opt. 2015, 17, 114012.

(46) Serrano-Montes, A. B.; Langer, J.; Henriksen-Lacey, M.; Jimenez de Aberasturi, D.; Solís, D. M.; Taboada, J. M.; Obelleiro, F.; 
Sentosun, K.; Bals, S.; Bekdemir, A.; Stellacci, F.; Liz-Marzán, L. M. J.

Phys. Chem. C 2016, 120, 20860-20868.

(47) Zhao, Y. H.; Luo, W. Q.; Kanda, P.; Cheng, H. W.; Chen, Y. Y.; Wang, S. P.; Huan, S. Y. Talanta 2013, 113, 7-13.

(48) Li, J. F.; Tian, X. D.; Li, S. B.; Anema, J. R.; Yang, Z. L.; Ding, Y.; Wu, Y. F.; Zeng, Y. M.; Chen, Q. Z.; Ren, B.; Wang, Z. L.; Tian, Z. Q. Nat. Protoc. 2013, 8, 52-65.

(49) Cai, H.; Zhu, J.; Chen, G.; Liu, L.; He, G. S.; Zhang, X. J. Raman Spectrosc. 2011, 42, 1722-1727.

(50) Li, Z. J.; Zhang, H. W.; Shen, J. S. Polym. Compos. 2011, 32, $1712-1717$.

(51) Kloust, H.; Schmidtke, C.; Merkl, J. P.; Feld, A.; Schotten, T.; Fittschen, U. E. A.; Gehring, M.; Ostermann, J.; Poselt, E.; Weller, H. J. Phys. Chem. C 2013, 117, 23244-23250. 